\title{
Interatrial septal thickness as a marker of structural and functional remodeling of the left atrium in patients with atrial fibrillation
}

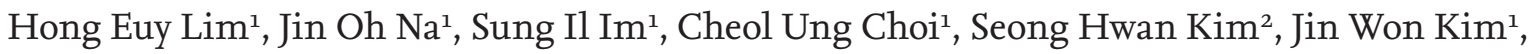

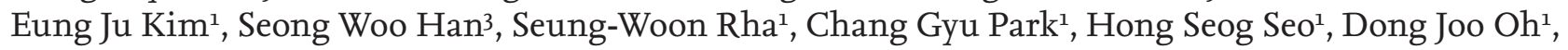 \\ and Chun Hwang 4
}

\author{
${ }^{1}$ Cardiovascular Center, Korea \\ University Guro Hospital, \\ Seoul; ' 2 Division of Cardiology, \\ Department of Internal Medicine, \\ Korea University Ansan Hospital, \\ Ansan; 3Division of Cardiology, \\ Department of Internal Medicine, \\ Hallym University Hangang \\ Sacred Heart Hospital, Seoul, \\ Korea; ${ }^{4}$ Division of Cardiology, \\ Utah Valley Regional Medical \\ Center, Provo, UT, USA
}

Background/Aims: There have been reports that interatrial septal (IAS) thickness is increased in patients with atrial fibrillation (AF). This study was performed to investigate whether IAS thickness determined by transthoracic echocardiography (TTE) represents the amount of left atrium (LA) structural and functional remodeling.

Methods: The study population consisted of 104 consecutive patients who underwent catheter ablation (CA) for AF (paroxysmal atrial fibrillation [PAF], 82; persistent atrial fibrillation [PeAF], 22). IAS thickness and left atrium volume (LAV) using TTE, and LA voltage (LAVOL) using 3-dimensional electroanatomical mapping system were assessed during sinus rhythm.

Results: IAS thickness was significantly correlated with maximal LAV (LAV $\max$ ) $(r=0.288, p=0.003)$, mean LAVOL $(r=-0.537, p<0.001)$, total left atrium emptying fraction $\left(\mathrm{LAEF}_{\text {total }} ; r=-0.213, p=0.030\right)$, and active LAEF $\left(\mathrm{LAEF}_{\text {active }} ; r=-0.249, p\right.$ $=0.014)$. IAS thickness was greater in the high-risk group $(\geq 2)$ compared to other groups according to $\mathrm{CHA}_{2} \mathrm{DS}_{2}$-VASc score $(p=0.019)$. During a follow-up of 19.6 months, 23 subjects (22.1\%; PAF, 17; PeAF, 6) had recurrence of arrhythmia. Univariate analysis showed that $\mathrm{LAV}_{\max }$, minimal LAV, mean $\mathrm{LA}_{\mathrm{VOL}}, \mathrm{LVEF}_{\text {total }}$, LVE-

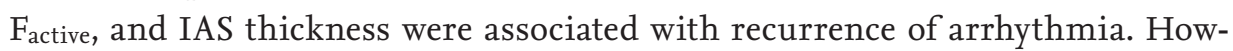
ever, on multivariate analysis, only mean LAvoL and LAEF $F_{\text {active }}$ were independent risk factors for recurrence.

Conclusions: Although IAS thickness showed significant correlations with parameters for LA structural and functional remodeling, this parameter alone could not independently predict recurrence of arrhythmia after CA for AF.

Keywords: Atrial fibrillation; Atrial septum; Heart atria; Atrial remodeling

\section{INTRODUCTION}

Atrial fibrillation (AF) is a major risk factor for ischemic stroke and is associated with increased morbidity and mortality [1]. Although catheter ablation (CA) for AF has emerged as an effective and curative treatment option
$[2,3]$, a substantial proportion of patients experience recurrence of arrhythmia after AF ablation [4,5].

Previous studies have indicated that structural remodeling of the left atrium (LA), such as atrial fibrosis as well as enlarged left atrium volume (LAV), is an independent risk factor for predicting AF recurrence af- 
ter CA [6-8]. The amount of LA scarring measured by three-dimensional $(3 \mathrm{D})$ voltage mapping is a powerful independent predictor of procedural outcome after CA [6]. Recent studies have suggested that delayed-enhancement magnetic resonance imaging (DE-MRI) may be a useful noninvasive modality to assess the amount of LA fibrosis, and to predict functional remodeling of LA and recurrence rate after AF ablation [7,9]. However, DE-MRI is not routinely available outside of research facilities. Therefore, more widely available and more reproducible noninvasive imaging techniques should be investigated for use in clinical practice.

There have been reports that interatrial septal (IAS) thickness was increased in patients with AF $[10,11]$. Moreover, we reported previously that IAS thickness was associated with amount of epicardial adipose tissue (EAT), which may be related to atrial arrhythmogenicity [12]. The present study was performed to determine whether IAS thickness is a simple, noninvasive imaging parameter that can be used to represent the degree of LA remodeling and to examine whether it may predict procedural outcome after $\mathrm{CA}$ in patients with $\mathrm{AF}$.

\section{METHODS}

\section{Study sample}

A total of 242 consecutive patients scheduled to undergo CA for AF at Korea University Cardiovascular Center between November 2009 and October 2010 were enrolled in this study. All enrolled subjects underwent transthoracic echocardiography (TTE), multi-slice computed tomography (MSCT), and contact LA voltage ( $\left.\mathrm{LA}_{\mathrm{VOL}}\right)$ mapping using a $3 \mathrm{D}$ electroanatomical mapping system (NavX, St. Jude Medical, Inc., Minneapolis, MN, USA) prior to $\mathrm{CA}$ on the same day. We excluded subjects who had AF at the time of echocardiographic examination and who required direct current (DC) cardioversion more than three times to restore sinus rhythm (SR) during $\mathrm{LA}_{\mathrm{VOL}}$ mapping. Other exclusion criteria were the presence of visible thrombi in LA on transesophageal echocardiography (TEE), previous AF ablation or cardiac surgery, aortic aneurysm or dissection, renal insufficiency, hyperthyroidism, electrolyte imbalance, alcohol abuse, malignancy, connective tissue disease, any acute or chronic inflammatory disease, and an implanted pacemaker or defibrillator. All antiarrhythmic drugs were discontinued at least five half-lives prior to examination. Amiodarone was discontinued $\geq 8$ weeks earlier. AF subjects were subdivided into paroxysmal atrial fibrillation (PAF) or persistent atrial fibrillation (PeAF) according to the most recent guidelines of the Heart Rhythm Society and European Cardiac Arrhythmia Society [13]. Enrolled subjects were divided into three groups according to $\mathrm{CHADS}_{2}$ score (o, low risk; 1, intermediate risk; $\geq 2$, high risk) and $\mathrm{CHA}_{2} \mathrm{DS}_{2}$-VASc score (o, low risk; 1 , intermediate risk; $\geq$ 2, high risk). Each participant signed an informed consent form prior to enrollment in the study, which was approved by the Human Subjects Review Committee of Korea University Hospital. This study was registered as KCTooooo79 at http://cris.nih.go.kr.

\section{Transthoracic echocardiography}

All subjects underwent two-dimensional, M-mode, Doppler, and tissue Doppler imaging echocardiography during SR. All examinations were performed using a commercially available Vivid 7 (GE Medical Systems, Vingmed, Horten, Norway) ultrasound system. All recorded echocardiograms were collected and analyzed using a computerized off-line analysis station (Echopa 6.3.4, GE Medical Systems).

All measurements were derived from three consecutive cardiac cycles and averaged. For each view, the transducer was carefully angled to quantitatively maximize atrial size, and gain positions were adjusted to obtain the clearest outline of the endocardium. Each echocardiographic parameter was determined according to the recommendations of the American Society of Echocardiography [14].

\section{Interatrial septal thickness assessment}

The maximal thickness of IAS was measured in apical four-chamber view after zooming in end-diastolic phase (Fig. 1A). To assess the tissue characteristics of IAS, the values of integrated backscatter(IBS) were obtained from the IAS and LA cavity in the apical four-chamber view by positioning the $5 \times 5 \mathrm{~mm}$ sample volume at each site while measuring IAS thickness. As there is no pericardium in the IAS, the IBS reflectivity of IAS was calculated as the ratio between the IBS values of IAS and the LA cavity $\times 100$. All IBS values were measured during the end-diastolic phase at the same phase of the cardiac 

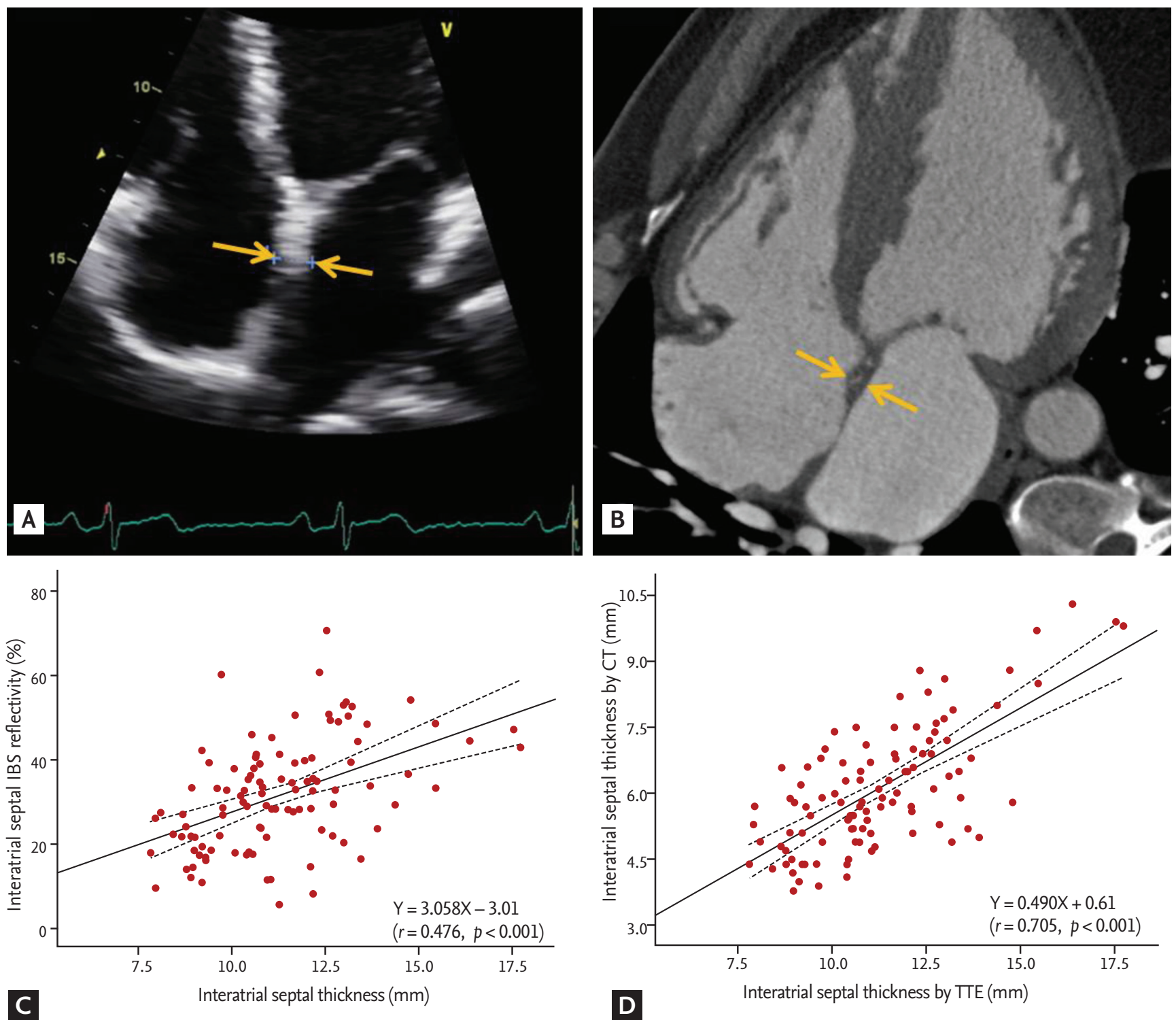

Figure 1. (A, B) Representative images of interatrial septal (IAS) thickness measurement by transthoracic echocardiography (TTE) and multi-slice computed tomography (MSCT). The IAS thickness measured by TTE showed good correlations with (C) integrated backscatter (IBS) reflectivity of the IAS and (D) IAS thickness measured by MSCT.

cycle. IAS thickness was also measured by MSCT (Fig. 1B). MSCT images were acquired using a 64-channel CT scanner (Brilliance 64, Philips Healthcare, Cleveland, $\mathrm{OH}, \mathrm{USA})$. Imaging was performed during a single inspiratory breath hold, in the craniocaudal direction, in helical mode, with retrospective electrocardiographic (ECG) gating. Axial multiphase images were reconstructed (slice thickness, $0.8 \mathrm{~mm}$; increment, $0.5 \mathrm{~mm}$ ) using retrospective ECG gating.

\section{LA function assessment}

LA anteroposterior dimension was obtained in the parasternal long axis view to assess the diameter of the LA. LAV was determined with the modified Simpson's method, as we reported previously [15]. To assess the LA phasic emptying fraction during the cardiac cycle, LAVs were measured at the mitral valve opening (maximal LAV, $\left.\mathrm{LAV}_{\text {max }}\right), \mathrm{LAV}$ at the onset of atrial emptying $\left(\mathrm{LAV}_{\mathrm{OAE}}, \mathrm{P}\right.$ wave onset of the electrocardiogram), and at mitral valve closure (minimal LAV, LAV $\mathrm{min}_{\text {min }}$ ) from the apical two- and four-chamber views. Detailed LA transport functions 
were calculated as follows:

Total LAEF $\left(\mathrm{LAEF}_{\text {total }}\right)=100 \times\left(\mathrm{LAV}_{\text {max }}-\mathrm{LAV}_{\text {min }}\right) / \mathrm{LAV}_{\max }$ Passive $\operatorname{LAEF}\left(\mathrm{LAEF}_{\text {passive }}\right)=100 \times\left(\mathrm{LAV}_{\max }-\mathrm{LAV}_{\mathrm{OAE}}\right) / \mathrm{LAV}_{\text {max }}$ Active $\operatorname{LAEF}\left(\mathrm{LAEF}_{\text {active }}\right)=100 \times\left(\mathrm{LAV}_{\mathrm{OAE}}-\mathrm{LAV}_{\text {min }}\right) / \mathrm{LAV}_{\mathrm{OAE}}$ LAEF, left atrium emptying fraction.

\section{Three-dimensional LA voltage mapping}

TEE and MSCT were performed immediately prior to CA in all subjects. After double transseptal puncture, systemic anticoagulation was achieved via intravenous heparin to maintain an activated clotting time between 300 and 350 seconds. After determination of the $3 \mathrm{D}$ geometry of the LA and pulmonary veins (PVs) using the NavX mapping system and merging with volume-rendered MSCT imaging, we performed high-density $3 \mathrm{D}$ voltage mapping using an open-irrigation, 3.5-mm-tip deflectable catheter (Celsius, Biosense Webster, Diamond Bar, CA, USA). In cases in which $\mathrm{AF}$ occurred during ${ }_{3} \mathrm{D}$ voltage mapping, internal DC cardioversion ( 5 to 15 J, biphasic shocks with R-wave synchronization, anodal decapolar catheter in the high right atrium to cathodal duodecapolar catheter inside the coronary sinus) (Lifepak 12, Physio-Control Ltd., Redmond, WA, USA) was conducted. Three-dimensional voltage maps were obtained during high right atrial pacing with a cycle length of $500 \mathrm{~ms}$. To evaluate regional voltage and ensure uniform distribution, LA was divided into six segments (posterior, roof, anterior, lateral, septal, and inferior wall) and then peak-to-peak bipolar voltages were obtained at more than 20 points in each segment. The mean voltage was determined by averaging the bipolar voltage of the points within the given region. An average of $174.7 \pm 24.0$ points (range, 135 to 214 ) were collected in the LA. Voltage mapping was not conducted in patients with reinitiating AF requiring DC cardioversion more than three times during the mapping procedure.

\section{Ablation procedures}

All PVs were mapped with a decapolar circular catheter (Lasso, Biosense Webster). Radiofrequency energy was delivered at a maximum power output of 25 to $30 \mathrm{~W}$, flow rate of 17 to $30 \mathrm{~mL} / \mathrm{min}$, and maximum temperature of $48^{\circ} \mathrm{C}$. The endpoint for each individual application at a given site was either total voltage abatement or current application of up to 40 seconds with adequate tissue contact and power delivery. A stepwise ablation procedure was performed in all AF patients regardless of AF type.
Initially, all patients underwent wide circumferential pulmonary vein isolation (CPVI). All ablated PVs were revisited after a waiting period of at least 30 minutes. PVs with evidence of reconnection were reisolated. For patients who remained in SR after CPVI, an inducibility test with $10 \mathrm{~mA}$ pacing was performed in the high right atrium or coronary sinus using a pacing cycle length of 180 ms with 1:1 capture. If induced AF was sustained for more than 10 minutes, additional ablation of the complex fractionated atrial electrogram (CFAE) sites on both atria was completed until AF termination was observed. CFAE was defined as electrograms with a fractionated interval between 50 and $120 \mathrm{~ms}$, displayed by color map on the NavX geometry. For patients who remained in AF after CPVI, CFAE ablation on both atria was also performed until AF termination was observed. In cases where AF converted to organized atrial flutter (AFL), a local activation time map was created during stable AFL, and was displayed on the NavX geometries of the atria. Several rounds of radiofrequency energy were then delivered to the critical isthmus or foci until AFL termination was observed. In patients with PeAF and/or induced typical AFL, a cavotricuspid isthmus line was created and bidirectional block was confirmed by a differential pacing maneuver. If subjects remained in AF up to 5 hours after transseptal puncture, SR was restored by internal DC cardioversion under isoproterenol infusion $(5 \mu \mathrm{g} / \mathrm{min})$. The procedural endpoints were:(1) no immediate reinitiation of AF after DC cardioversion, and (2) noninducibility of sustained organized AFL.

\section{Follow-up}

If no complications arose during the procedures, subjects were started on warfarin anticoagulation therapy without any antiarrhythmic medications. All enrolled subjects were prospectively followed up for recurrence of AF after CA via monthly outpatient clinic visits. Subjects also underwent 48-hour Holter monitoring at 1, 3, 6, and 12 months post-CA. Electrocardiography was performed at each visit and any time the subjects reported palpitations. In addition, subjects were questioned by telephone by a nurse practitioner at 2-week intervals and were instructed to call whenever they experienced symptoms. If any instances of AF or AFL were documented during the follow-up period, the subject was diagnosed with clinical recurrence of arrhythmia and antiarrhythmic medica- 
tions were prescribed.

\section{Statistical analyses}

All continuous variables are expressed as the mean \pm standard deviation or median (interquartile range) depending on the distribution. For continuous data, the statistical significance of differences was evaluated using Student $t$ test or Mann-Whitney $U$ test depending on the data distribution. Categorical variables are presented as fre- quencies (percentage) and the chi-square test was used for analysis of categorical variables. All correlations were examined using Spearman rank correlation test. The statistical significance of each group was assessed using the Kruskal-Wallis test for the $\mathrm{CHADS}_{2}$ score group and oneway ANOVA for the $\mathrm{CHA}_{2} \mathrm{DS}_{2}$-VASc score group. To determine whether any of the variables were independently related to recurrence of arrhythmia after CA, multivariate analysis of variables with $p<0.05$ on univariate analysis

Table 1. Baseline characteristics, echocardiographic measurements, and mean voltage of the left atrium

\begin{tabular}{|c|c|c|c|c|}
\hline Variable & Overall (n = 104) & $\operatorname{PAF}(\mathrm{n}=82)$ & $\operatorname{PeAF}(\mathrm{n}=22)$ & $p$ value $^{\mathrm{a}}$ \\
\hline Age, yr & $53.5 \pm 12.1$ & $54.3 \pm 11.7$ & $50.6 \pm 13.5$ & 0.252 \\
\hline Sex, male:female & $79: 25$ & $61: 21$ & $18: 4$ & 0.469 \\
\hline Body mass index, kg/m² & $24.6 \pm 2.7$ & $24.8 \pm 2.76$ & $24.1 \pm 2.50$ & 0.306 \\
\hline Diabetes & $11(10.6)$ & $8(9.8)$ & $3(13.6)$ & 0.599 \\
\hline Hypertension & $42(40.4)$ & $33(40.2)$ & $9(40.9)$ & 0.955 \\
\hline Systolic blood pressure, $\mathrm{mmHg}$ & $129.2 \pm 16.8$ & $129 \cdot 5 \pm 16.8$ & $128.1 \pm 17.1$ & 0.741 \\
\hline Diastolic blood pressure, $\mathrm{mmHg}$ & $75.6 \pm 10.2$ & $76.0 \pm 9.9$ & $74.0 \pm 11.1$ & 0.445 \\
\hline Heart rate, beat/min & $65.9 \pm 11.9$ & $66.4 \pm 11.0$ & $64 \cdot 3 \pm 15 \cdot 0$ & 0.541 \\
\hline C-reactive protein, mg/dL & $2.05 \pm 3.69$ & $1.79 \pm 2.85$ & $2.90 \pm 5.65$ & 0.447 \\
\hline Total cholesterol, mg/dL & $173 \cdot 5 \pm 32.8$ & $175 \cdot 3 \pm 33.8$ & $167.0 \pm 28.7$ & 0.252 \\
\hline Triglyceride, mg/dL & $108(73-159)$ & $116(72-161)$ & $103(75-136)$ & 0.566 \\
\hline HDL-C, mg/dL & $47.1 \pm 10.9$ & $47.1 \pm 10.9$ & $47.1 \pm 11.0$ & 0.985 \\
\hline LDL-C, mg/dL & $109.1 \pm 28.4$ & $111.1 \pm 29.1$ & $102.0 \pm 25.0$ & 0.156 \\
\hline $\mathrm{CHADS}_{2}$ score & $0.71 \pm 0.89$ & $0.68 \pm 0.89$ & $0.82 \pm 0.91$ & 0.469 \\
\hline $\mathrm{CHA}_{2} \mathrm{DS}_{2}$-VASc score & $1.16 \pm 1.12$ & $1.16 \pm 1.09$ & $1.18 \pm 1.26$ & 0.901 \\
\hline \multicolumn{5}{|l|}{ Echocardiography } \\
\hline LVEF, \% & $65 \cdot 4 \pm 7.7$ & $65.8 \pm 6.7$ & $63.7 \pm 10.8$ & 0.376 \\
\hline LA dimension, $\mathrm{mm}$ & $40.4 \pm 5.8$ & $40.2 \pm 5.8$ & $41.3 \pm 6.0$ & 0.452 \\
\hline $\mathrm{LAV}_{\max }, \mathrm{mL}$ & $69.1 \pm 19.6$ & $66.8 \pm 18.4$ & $77.8 \pm 21.8$ & 0.039 \\
\hline $\mathrm{LAV}_{\mathrm{OAE}}, \mathrm{mL}$ & $51.8 \pm 18.2$ & $50.2 \pm 17.5$ & $57.5 \pm 20.0$ & 0.135 \\
\hline $\mathrm{LAV}_{\min }, \mathrm{mL}$ & $38.7 \pm 15 \cdot 3$ & $37.2 \pm 14.8$ & $44.4 \pm 16.0$ & 0.064 \\
\hline $\mathrm{LAEF}_{\text {total }}, \%$ & $44.7 \pm 9.7$ & $45 \cdot 1 \pm 9 \cdot 3$ & $43.4 \pm 11.0$ & 0.530 \\
\hline LAEF $_{\text {passive }}, \%$ & $26.0 \pm 10.0$ & $25.7 \pm 10.4$ & $26.9 \pm 8.9$ & 0.617 \\
\hline $\mathrm{LAEF}_{\text {active }}, \%$ & $26.0 \pm 9.1$ & $26.4 \pm 9.5$ & $24.6 \pm 7.5$ & 0.355 \\
\hline IAS thickness, mm & $11.26 \pm 2.02$ & $11.07 \pm 1.93$ & $11.95 \pm 2.21$ & 0.068 \\
\hline Mean LAvoL, mV & $2.34 \pm 1.05$ & $2.45 \pm 1.08$ & $1.95 \pm 0.84$ & 0.024 \\
\hline
\end{tabular}

Values are presented as mean $\pm \mathrm{SD}$, number (\%), or median (interquartile range).

PAF, paroxysmal atrial fibrillation; PeAF, persistent atrial fibrillation; HDL-C, high density lipoprotein cholesterol; LDL-C, low density lipoprotein cholesterol; LVEF, left ventricular ejection fraction; LA, left atrium; LAV $V_{\max }$, maximal left atrial volume; $\mathrm{LAV}_{\mathrm{OAE}}$, left atrial volume at the onset of atrial emptying; $\mathrm{LAV}_{\min }$, minimal left atrial volume; $\mathrm{LAEF}_{\text {total, }}$ total left atrial emptying fraction; $\mathrm{LAEF}_{\text {passive, }}$ passive left atrial emptying fraction; $\mathrm{LAEF}_{\mathrm{active}}$, active left atrial emptying fraction; IAS, interatrial septum; LAvol, left atrial voltage.

ap value between paroxysmal and persistent atrial fibrillation groups. 
was performed using linear regression analysis. The area under the receiver operating characteristic (ROC) curve was used to evaluate the accuracy of prediction of recurrence, and then to derive sensitivity and specificity values for each cutoff value. Finally, to test the reproducibility of the IAS thickness measurement, inter- and intraobserver variability were analyzed. Bland-Altman analysis was performed to express reproducibility of measurements for IAS thickness between each investigator. All statistical analyses were conducted using SPSS version 13.0 (SPSS Inc., Chicago, IL, USA). Statistical significance was set at $p<0.05$ (two-sided).
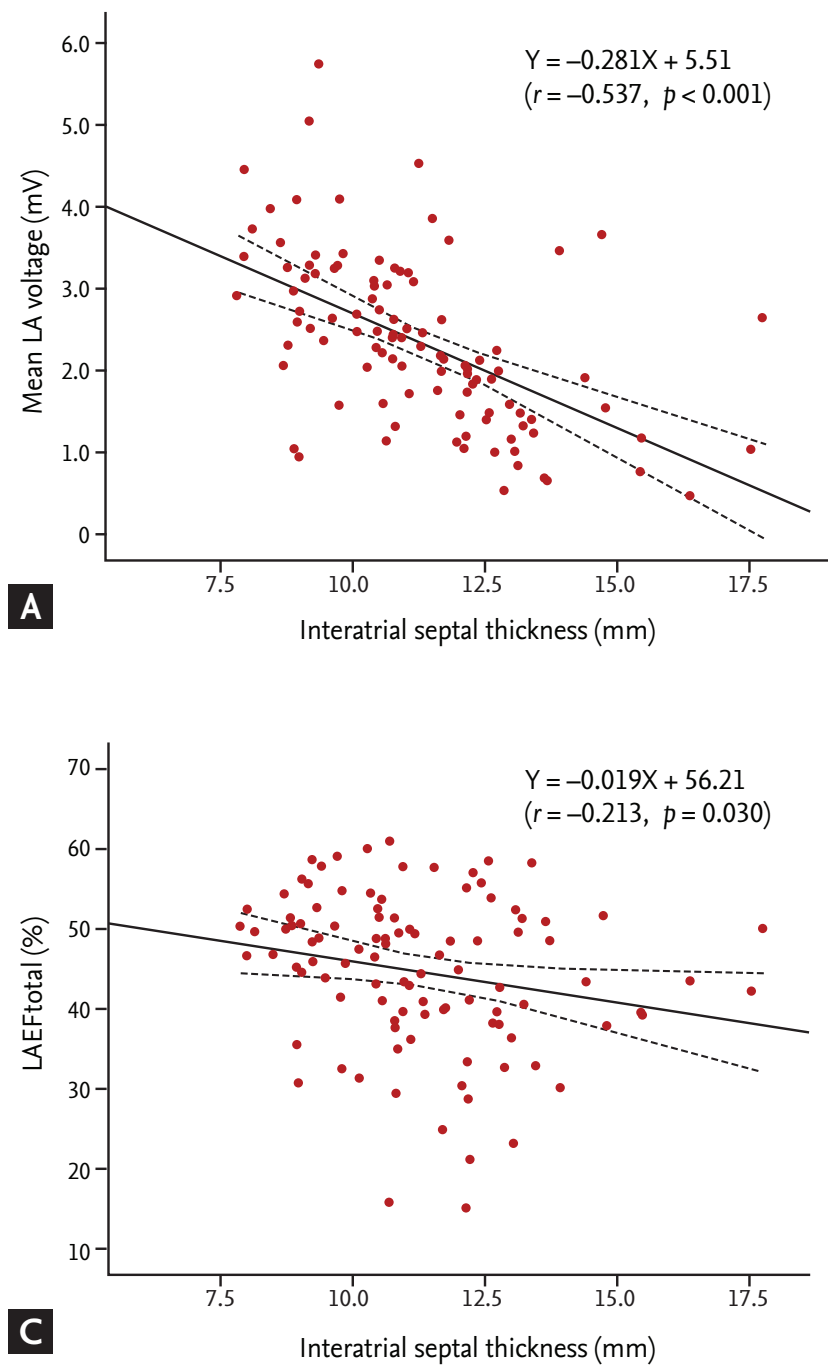

\section{RESULTS}

Of the initial total of 242 consecutive patients, 116 were excluded because they had AF at the time of T'TE examination and 22 were excluded because they required DC cardioversion more than three times during $\mathrm{LA}_{\mathrm{VOL}}$ mapping. Therefore, a total of 104 subjects were enrolled in this study.

The baseline characteristics of all enrolled subjects are summarized in Table 1. The mean age was $53.5 \pm 12.1$ years, 79 subjects $(76 \%)$ were male, 82 subjects had PAF, and 22 had PeAF. The mean $\mathrm{CHADS}_{2}$
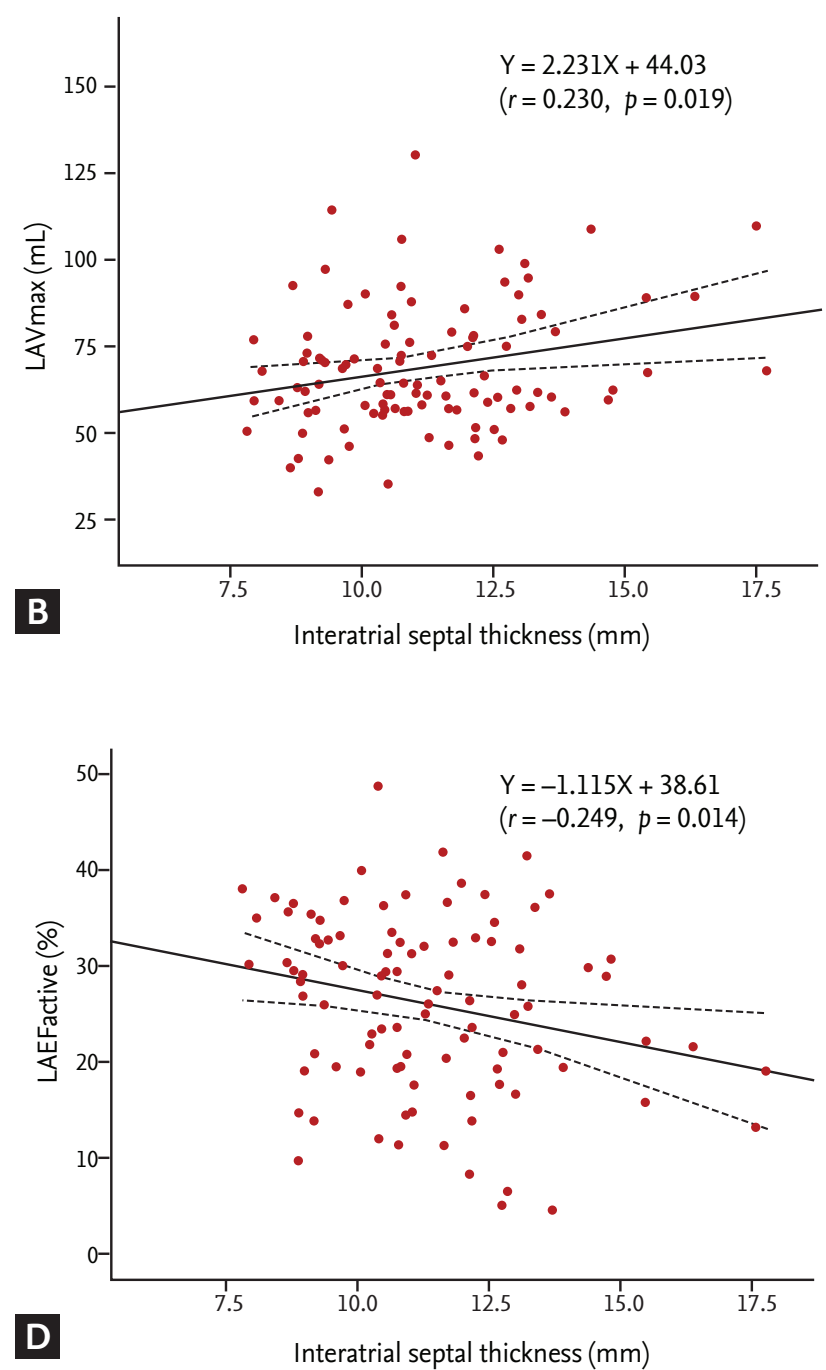

Figure 2. Relationships between interatrial septal (IAS) thickness and left atrial (LA) structural and functional parameters. (A) Linear regression between IAS thickness and mean LA voltage. (B) Linear regression between IAS thickness and maximal LAV $\left(\mathrm{LAV}_{\max }\right)$. (C) Linear regression between IAS thickness and total left atrial emptying fraction (LAEF $\left.F_{\text {total }}\right)$. (D) Linear regression between IAS thickness and active $\mathrm{LAEF}\left(\mathrm{LAEF}_{\text {active }}\right)$. 
score was $0.71 \pm 0.89$ and mean $\mathrm{CHA}_{2} \mathrm{DS}_{2}$-VASc score was $1.16 \pm 1.12$. Mean IAS thickness was $11.26 \pm 2.02 \mathrm{~mm}$. $\mathrm{LAV}_{\max }$ was significantly greater and mean $\mathrm{LA}_{\mathrm{VOL}}$ was lower in the PeAF group than in the PAF group. The IBS reflectivity of IAS showed a positive correlation with IAS thickness $(r=0.476, p<0.001)$ (Fig. $1 \mathrm{C})$. Moreover, IAS thickness measured by TTE was closely correlated with that determined by MSCT $(r=0.705, p<0.001)$ (Fig. 1D).

Mean $\mathrm{LA}_{\mathrm{VOL}}$ and septal $\mathrm{LA}_{\mathrm{VOL}}$ showed negative correlations with IAS thickness $(r=-0.537, p<0.001 ; r=-0.330$, $p=0.001$, respectively). In addition, mean $L A_{V O L}$ showed positive correlations with $\mathrm{LAEF}_{\text {total }}$ and $\mathrm{LAEF}_{\text {active }}(r=0.292$, $p=0.003 ; r=0.283, p=0.005$, respectively). IAS thickness was associated with LAVs and LAEFs (Fig. 2). It was also correlated with $\mathrm{CHA}_{2} \mathrm{DS}_{2}$-VASc score $(r=0.348, p<0.001)$

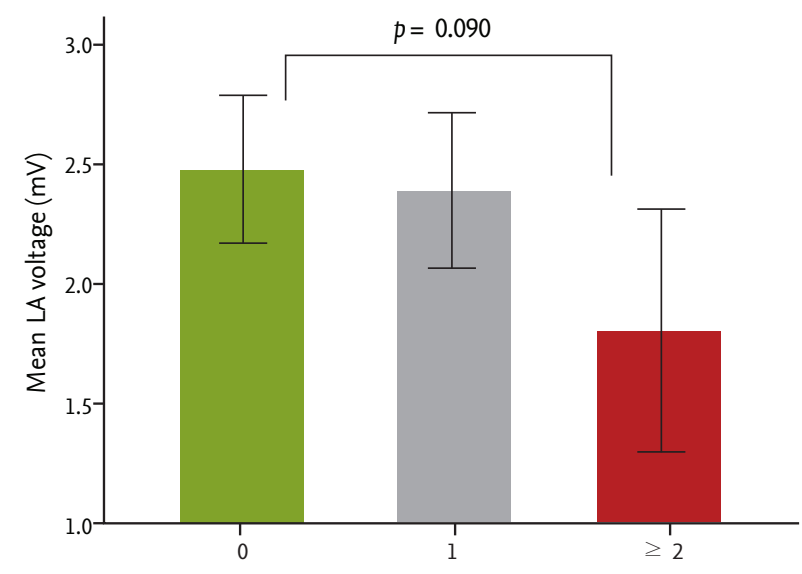

A

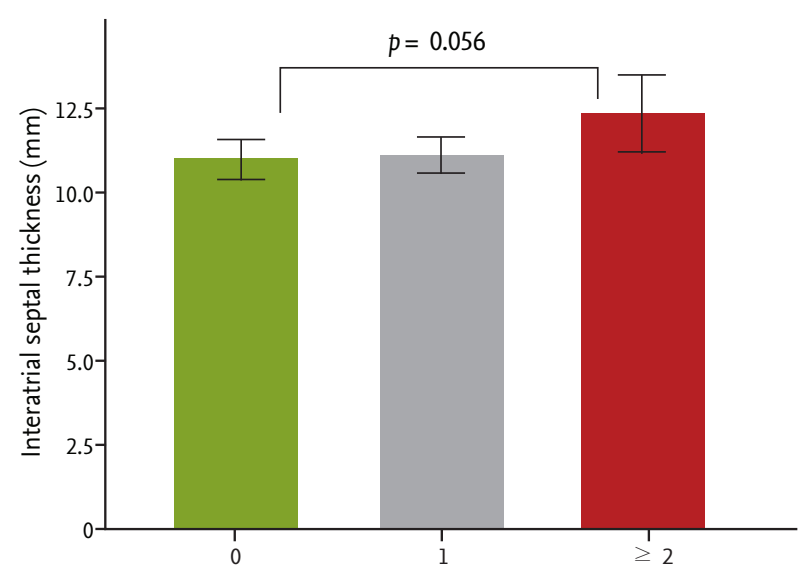

C

$\mathrm{CHADS}_{2}$ score and $\mathrm{CHADS}_{2}$ score $(r=0.208, p=0.034)$. Fig. 3 shows a comparison of mean $\mathrm{LA}_{\mathrm{VOL}}$ and IAS thickness among three groups according to $\mathrm{CHADS}_{2}$ score $(\mathrm{o}, \mathrm{n}=52 ; 1$, $\mathrm{n}=36 ; \geq 2, \mathrm{n}=16)$, and $\mathrm{CHA}_{2} \mathrm{DS}_{2}$-VASc score $(\mathrm{o}, \mathrm{n}=35$; $1, \mathrm{n}=34 ; \geq 2, \mathrm{n}=35)$. Mean $\mathrm{LA} \mathrm{VOL}_{\mathrm{L}}$ was much lower in the high-risk group than in the other groups according to $\mathrm{CHADS}_{2}$ score $(p=0.090)$ (Fig. $\left.3 \mathrm{~A}\right)$ and $\mathrm{CHA}_{2} \mathrm{DS}_{2}$-VASc score $(p=0.001$ ) (Fig. $3 B$ ). IAS thickness was much greater in the high-risk group than the other groups according to $\mathrm{CHADS}_{2}$ score $(p=0.056)$ (Fig. $\left.3 \mathrm{C}\right)$ and $\mathrm{CHA}_{2} \mathrm{DS}_{2}$-VASc score $(p=0.019)$ (Fig. 3D).

The clinical and laboratory findings influencing AF recurrence are listed in Table 2. During the mean follow-up of $19.6 \pm 4.1$ months after a single ablation procedure, 23 subjects (22.1\%; PAF, 17; PeAF, 6) had recurrence
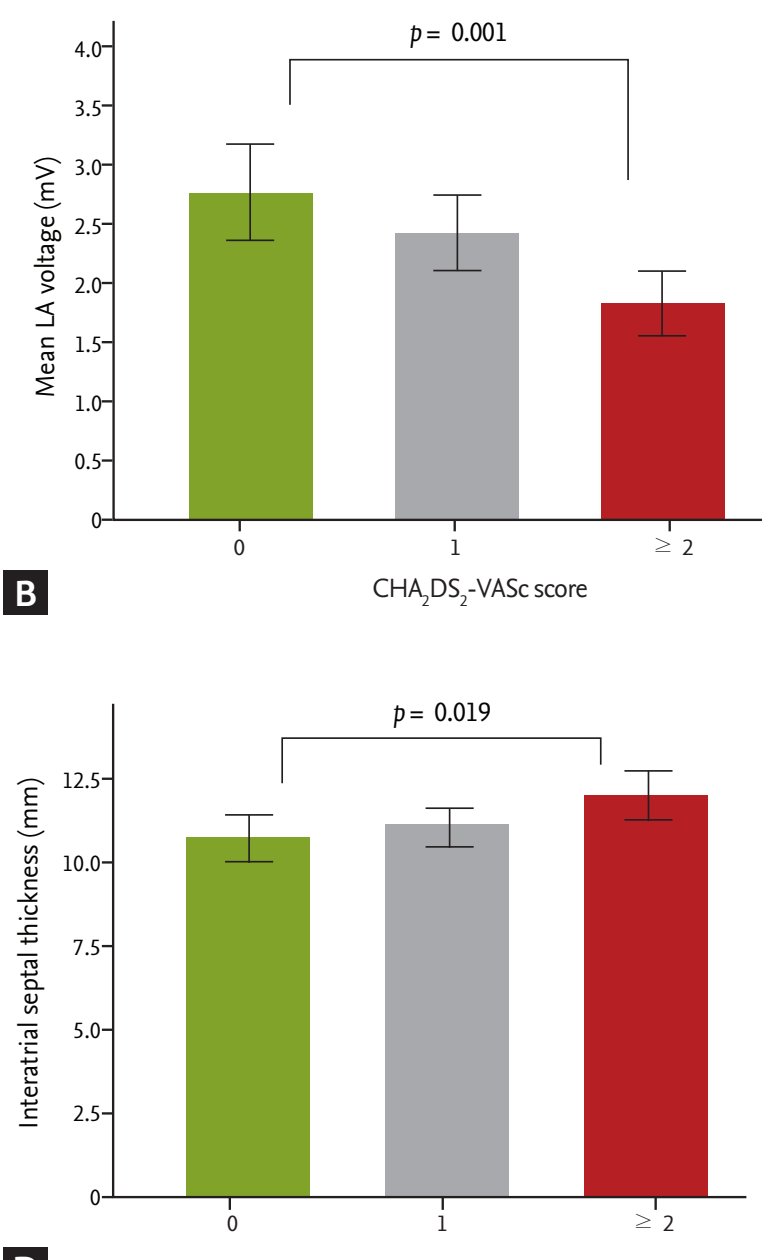

D

$\mathrm{CHA}_{2} \mathrm{DS}_{2}$-VASc score

Figure 3. Mean left atrial voltage (LAvoL) and interatrial septal (IAS) thickness according to $\mathrm{CHADS}_{2} \mathrm{Score}_{\mathrm{and}} \mathrm{CHA}_{2} \mathrm{DS}_{2}-\mathrm{VASc}$

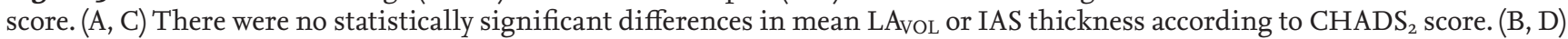
However, mean LAvol was significantly lower and IAS thickness was significantly higher in high-risk $\mathrm{CHA}_{2} \mathrm{DS}_{2}$-VASc level $(\geq 2)$. 
of arrhythmia. Univariate analysis showed that phasic LAVs, $\mathrm{LAEF}_{\text {total }}, \mathrm{LAEF}_{\text {active }}$, IAS thickness, and mean LAvoL were associated with AF recurrence. Multivariate analysis revealed that $\mathrm{LAEF}_{\text {active }}$ and mean $\mathrm{LA}_{\mathrm{VOL}}$ were independent risk factors for AF recurrence after CA.

Fig. 4 shows the ROC curves for mean $\mathrm{LA}_{\mathrm{VOL}}$ and LAE$\mathrm{F}_{\text {active }}$ for prediction of AF recurrence. Mean $\mathrm{LA}_{\mathrm{VOL}}<2.01$ $\mathrm{mV}$ showed sensitivity of $73.9 \%$ and specificity was $71.6 \%$ ( $p<0.001)$. Using a cutoff value of $23.5 \%$ in $\mathrm{LAEF}_{\text {active, the }}$ sensitivity and specificity were $66.7 \%$ and $63.6 \%$, respectively $(p=0.003)$.

Fig. 5 shows the reproducibility of IAS thickness measurement. The correlation of intraobserver measurements was excellent $(r=0.821, p<0.001)$, and the correlation of interobserver measurements was good $(r=0.560$, $p=0.001)$. The Bland-Altman plot showed excellent reproducibility of IAS thickness measurement between investigators.

\section{DISCUSSION}

The most noteworthy findings of this study were: (1) IAS thickness measured by TTE correlated with the degree of structural remodeling (mean LA $\mathrm{VOL}_{\mathrm{L}}$ and LAVs) and functional remodeling $\left(\mathrm{LAEF}_{\text {total }}\right.$ and $\left.\mathrm{LAEF}_{\text {active }}\right)$ of the LA; (2) IAS thickness is associated with clinical surrogate parameters for LA remodeling, such as $\mathrm{CHADS}_{2}$ score and $\mathrm{CHA}_{2} \mathrm{DS}_{2}$-VASc score; and (3) IAS thickness, however, could not independently distinguish high-risk patients who are prone to recurrence of arrhythmia before the AF ablation procedure.

Table 2. Binary logistic regression analysis: variables influencing arrhythmia recurrence after catheter ablation for AF

\begin{tabular}{|c|c|c|c|c|}
\hline \multirow{2}{*}{ Variable } & \multicolumn{2}{|c|}{ AF recurrence } & \multirow{2}{*}{$\begin{array}{c}\text { Univariate } \\
\text { analysis }\end{array}$} & \multirow{2}{*}{$\begin{array}{c}\text { Multivariate } \\
\text { analysis }\end{array}$} \\
\hline & No $(\mathrm{n}=81)$ & Yes $(n=23)$ & & \\
\hline Age, yr & $52.7 \pm 12.3$ & $56.3 \pm 11.2$ & 0.191 & \\
\hline Female sex & $16(19.8)$ & $9(39.1)$ & 0.055 & \\
\hline Body mass index, $\mathrm{kg} / \mathrm{m}^{2}$ & $24.6 \pm 2.9$ & $24.6 \pm 1.9$ & 0.862 & \\
\hline Persistent atrial fibrillation & $16(19.8)$ & $6(26.1)$ & 0.512 & \\
\hline Systolic blood pressure, $\mathrm{mmHg}$ & $125.7 \pm 16.7$ & $127 \cdot 4 \pm 16.0$ & 0.837 & \\
\hline Diastolic blood pressure, $\mathrm{mmHg}$ & $75 \cdot 7 \pm 10.6$ & $75 \cdot 3 \pm 9.0$ & 0.848 & \\
\hline $\mathrm{CHADS}_{2}$ score & $0.64 \pm 0.87$ & $0.96 \pm 0.93$ & 0.155 & \\
\hline $\mathrm{CHA}_{2} \mathrm{DS}_{2}$-VASc score & $1.05 \pm 1.11$ & $1.57 \pm 1.12$ & 0.059 & \\
\hline \multicolumn{5}{|l|}{ Echocardiography } \\
\hline LVEF, \% & $65.5 \pm 8.0$ & $65.0 \pm 6.9$ & 0.756 & \\
\hline LA dimension, $\mathrm{mm}$ & $40.0 \pm 5.5$ & $41.9 \pm 6.7$ & 0.217 & \\
\hline $\mathrm{LAV}_{\max }, \mathrm{mL}$ & $66.0 \pm 17.4$ & $80.4 \pm 22.9$ & 0.009 & \\
\hline $\mathrm{LAV}_{\mathrm{OAE}}, \mathrm{mL}$ & $48.8 \pm 16.1$ & $61.8 \pm 21.7$ & 0.010 & \\
\hline $\mathrm{LAV}_{\min }, \mathrm{mL}$ & $35 \cdot 5 \pm 11.8$ & $50.0 \pm 20.5$ & 0.002 & \\
\hline $\mathrm{LAEF}_{\text {total }}, \%$ & $46.4 \pm 9.0$ & $38.7 \pm 9.8$ & 0.002 & \\
\hline $\mathrm{LAEF}_{\text {passive }}, \%$ & $26.7 \pm 10.2$ & $23.4 \pm 9.3$ & 0.159 & \\
\hline LAEF $_{\text {active }}, \%$ & $27.5 \pm 8.8$ & $21.0 \pm 8.8$ & 0.005 & 0.028 \\
\hline IAS thickness, mm & $11.0 \pm 1.9$ & $12.3 \pm 2.2$ & 0.014 & \\
\hline Mean LAvoL, mV & $2.55 \pm 1.03$ & $1.62 \pm 0.79$ & $<0.001$ & 0.011 \\
\hline
\end{tabular}

Values are presented as mean \pm SD or number (\%).

$\mathrm{AF}$, atrial fibrillation; LVEF, left ventricular ejection fraction; LA, left atrium; LAV $\max$, maximal left atrial volume; LAV atrial volume at the onset of atrial emptying; $\mathrm{LAV}_{\min }$, minimal left atrial volume; $\mathrm{LAEF}_{\text {total, }}$ total left atrial emptying fraction;

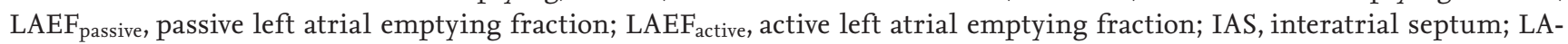
voL, left atrial voltage. 

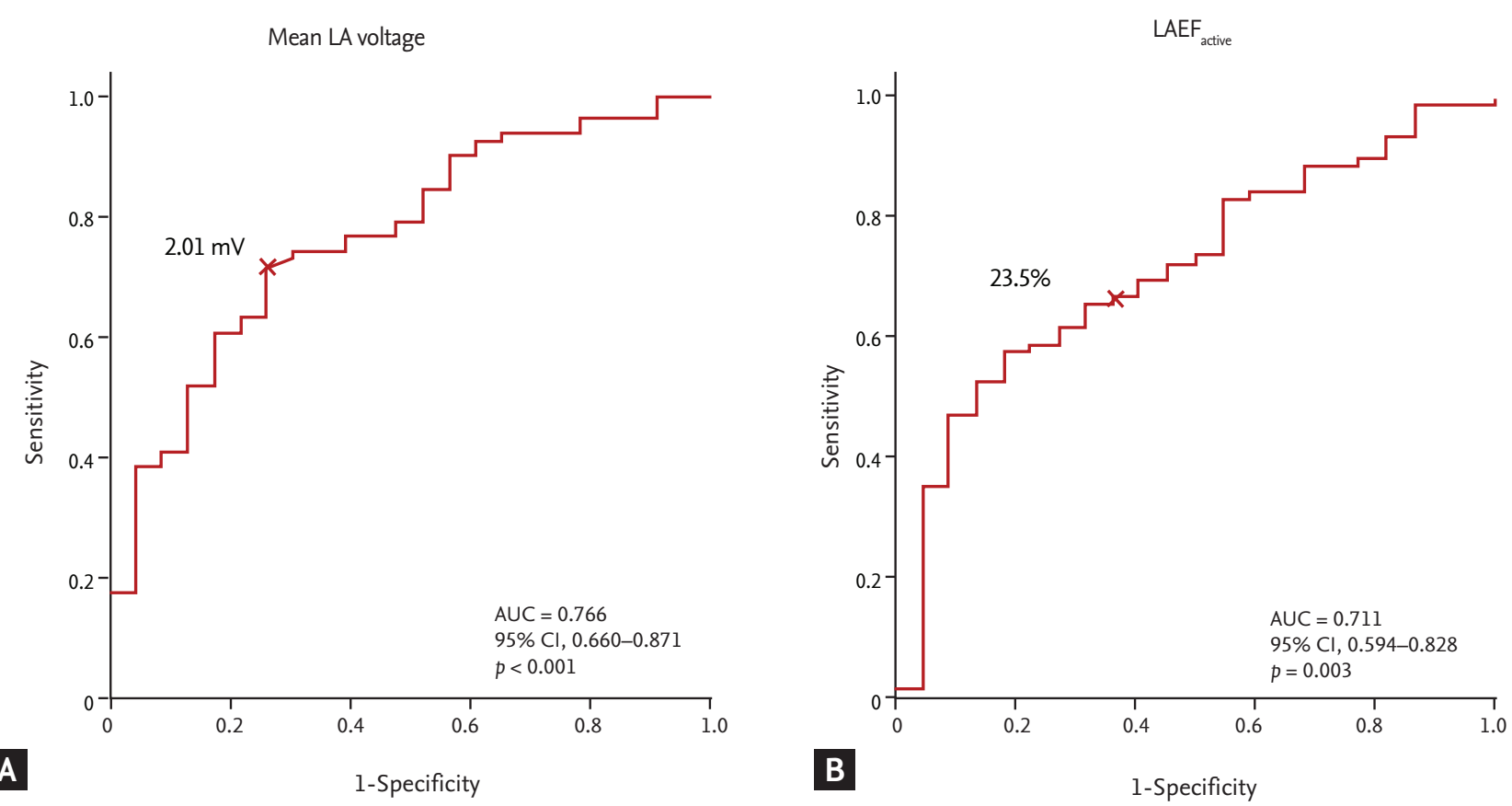

Figure 4. Receiver operator characteristic curves for (A) mean left atrial (LA) voltage and (B) active left atrial emptying fraction $\left(\mathrm{LAEF}_{\text {active }}\right)$ were used to distinguish subjects who are prone to recurrence of arrhythmia after atrial fibrillation ablation. AUC, area under the curve; CI, confidence interval.

Preexisting LA scarring assessed by $3 \mathrm{D}$ voltage mapping is a reliable independent predictor of procedural outcome [6]. Recently, DE-MRI was suggested as being useful for quantitative assessment of LA fibrosis, and providing a noninvasive modality for predicting functional remodeling of LA and recurrence rate after AF ablation $[7,9]$. However, these approaches to assess the amount of LA scarring are extremely labor-intensive and time-consuming. In addition, these modalities are not routinely available outside research facilities. In this regard, the findings of the present study have potential clinical utility because IAS thickness can be easily, reproducibly, and accurately determined by TTE.

Several recent studies indicated that EAT is associated with the prevalence and chronicity of AF, independently of other traditional risk factors $[16,17]$. Several possible mechanisms are likely to account for the pathogenic relationship between EAT and AF, including electrical and structural remodeling of the atria via proinflammatory properties. We also demonstrated that periatrial EAT, as well as the total volume of EAT, are significantly larger in AF subjects, as compared to those of matched controls [12]. Moreover, IAS thickness measured by MSCT was independently associated with LAV in patients with
AF. Previously, Lopez-Candales et al. [10,11] reported that IAS thickness measured by TTE and TEE was significantly increased in AF patients, and suggested that it might be a surrogate marker of changes in components within the atrial wall. As the posterior portion of IAS is essentially an extracardiac structure containing abundant adipose tissue [18-20], many investigators have suggested that atrial arrhythmia may be related to IAS thickness, and the mechanism of atrial arrhythmia may involve fatty tissue infiltration that interferes with the architecture of atrial myocytes [21-23]. In the present study, we showed that IAS thickness assessed by TTE was significantly correlated with IBS reflectivity of IAS, suggesting that the main determinant of IAS was EAT. Furthermore, we demonstrated that IAS thickness measured by TTE was closely associated with mean LA $A_{\mathrm{VOL}}$ and LAV.

The $\mathrm{CHADS}_{2}$ score, which has been used as a guideline for the prevention of ischemic stroke in $\mathrm{AF}$ patients, may be a useful clinical surrogate parameter reflecting the degree of LA remodeling. Daccarett et al. [24] reported that higher $\mathrm{CHADS}_{2}$ score was associated with a greater degree of LA scarring detected by DE-MRI. Chao et al. [25] suggested that higher $\mathrm{CHADS}_{2}$ scores 


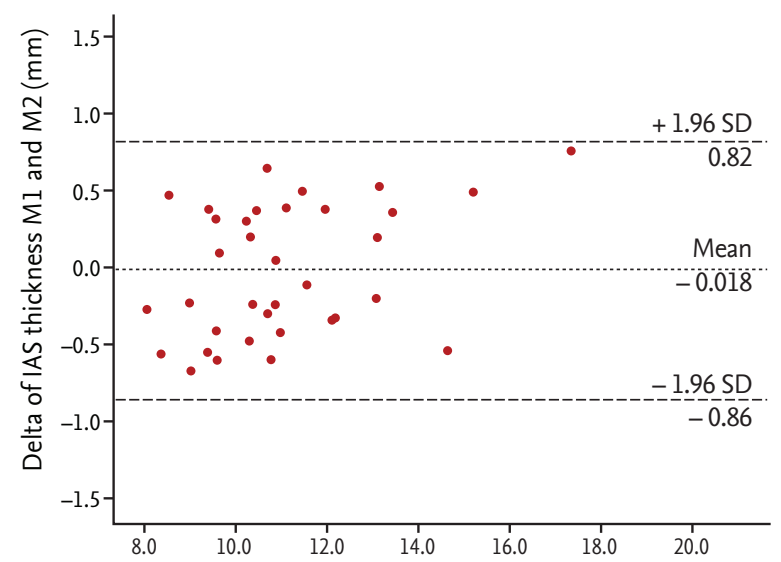

A

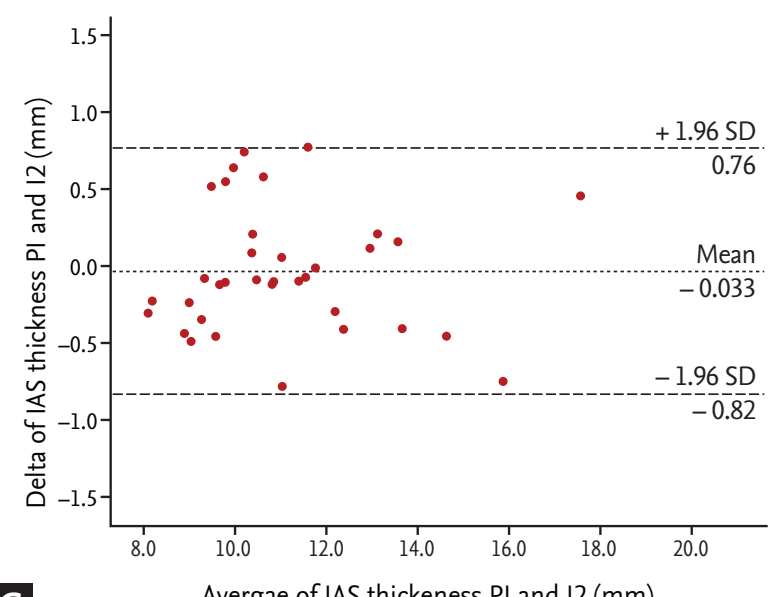

C

Avergae of IAS thickeness $\mathrm{PI}$ and $12(\mathrm{~mm})$
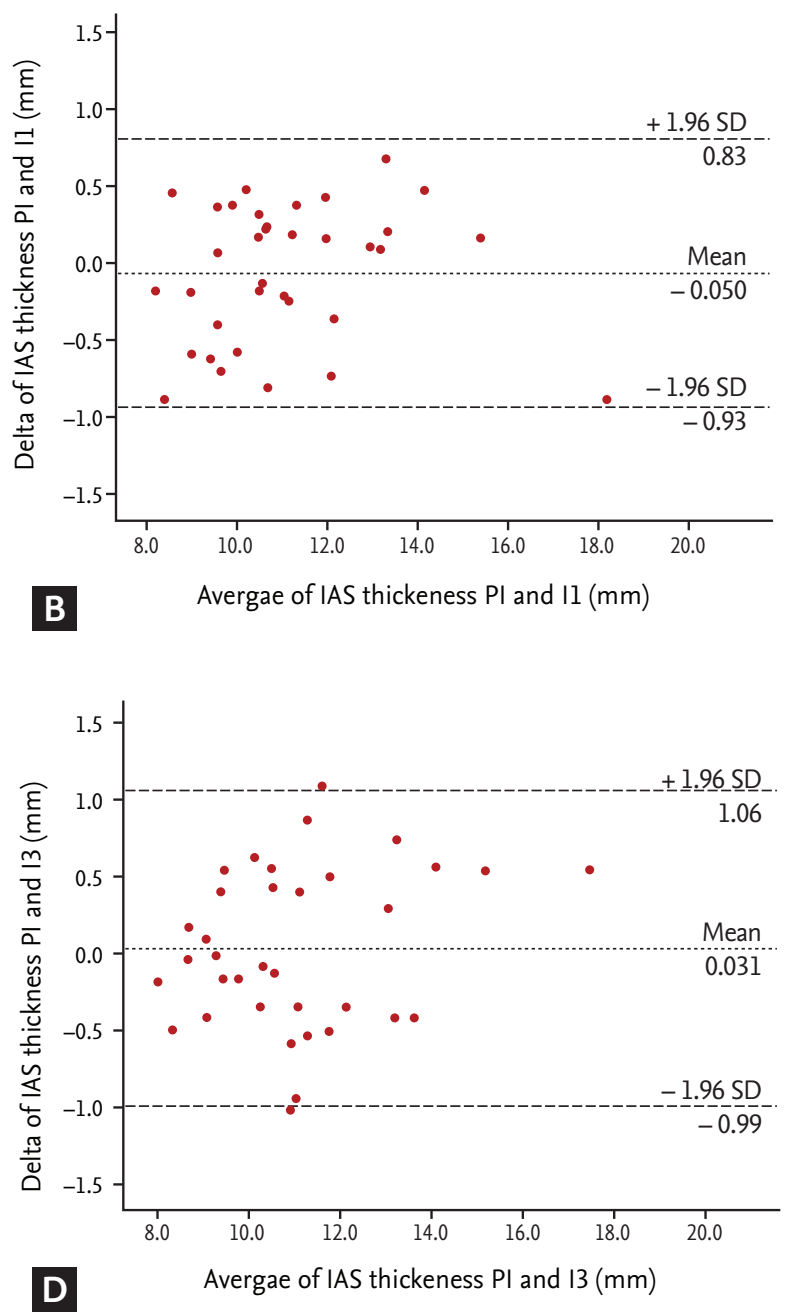

Figure 5. (A-D) Intra- and interobserver reproducibility of interatrial septal (IAS) thickness on transthoracic echocardiography. The Bland-Altman plot shows the excellent reproducibility of IAS thickness measurement between investigators.

may predict procedural outcome after AF ablation. Recently, the European Society of Cardiology suggested new guidelines for AF management [26] and proposed a $\mathrm{CHA}_{2} \mathrm{DS}_{2}$-VASc score, which may be more accurate for determining the intermediate-risk group than $\mathrm{CHADS}_{2}$ score. Our findings support the hypothesis that the $\mathrm{CHA}_{2} \mathrm{DS}_{2}$-VASc score is more closely related to the degree of LA structural remodeling than the $\mathrm{CHADS}_{2}$ score. IAS thickness was also more closely associated with the $\mathrm{CHA}_{2} \mathrm{DS}_{2}$-VASc score than the CHADS 2 score.

In the present study, we found that IAS thickness was significantly correlated with $\mathrm{LAEF}_{\text {total }}$ and $\mathrm{LAEF}_{\text {active }}$. As atrial mechanical contractile remodeling has generally been considered to be a major cause of thrombus formation in patients with AF [27-29], our findings suggest that IAS thickness may be a useful surrogate predictor of future thromboembolic risk in patients with AF. However, we cannot conclude that IAS thickness is an independent predictor for ischemic stroke because we did not investigate the occurrence rate of thromboembolic events in this study.

Finally, as a substantial number of patients experience recurrence of arrhythmia after AF ablation, assessment of the degree of LA structural and functional remodeling may be needed for patient selection prior to CA. From the this viewpoint, our findings are significant because IAS thickness allows identification of LA remodeling, but not high-risk patients who are prone to recurrence of arrhythmia after AF ablation. However, it could be assessed easily, noninvasively, and reproducibly as a means of determining LA remodeling. Therefore, IAS thickness may be a useful parameter for predicting 
the degree of LA structural and functional remodeling in patients with $\mathrm{AF}$.

In limitations, first, we enrolled only highly selected patients with SR at the time of examination because LAVOL mapping and $\mathrm{LAEF}_{\text {active }}$ should be assessed during SR. As we may have excluded patients with profound LA remodeling in this study, our results cannot be extrapolated to patients with long-lasting PeAF or permanent AF. Second, we cannot confirm that the main determinant of IAS was adipose tissue, although IAS thickness was positively correlated with IBS reflectivity and negatively correlated with septal $\mathrm{LA}_{\mathrm{VOL}}$. Finally, it is not clear whether adipose tissue located in the IAS itself induces LA remodeling or whether the thickening of IAS is a consequence of the LA remodeling process caused by other factors. Therefore, further investigations are warranted to determine whether there is a causal relationship between IAS thickness and LA remodeling.

In conclusion, measurement of IAS thickness may be a reliable, noninvasive parameter for reflecting the degree of LA structural and functional remodeling. Furthermore, as IAS thickness was closely associated with $\mathrm{CHA}_{2} \mathrm{DS}_{2}$-VASc score, this measure may be applied to future thromboembolic risk stratification in patients with AF. However, it was not a useful parameter to identify patients who are prone to recurrence of arrhythmia after AF ablation.

\section{KEY MESSAGE}

1. Interatrial septal (IAS) thickness is correlated with the degree of left atrium structural and functional remodeling.

2. IAS thickness is also associated with $\mathrm{CHADS}_{2}$ and $\mathrm{CHA}_{2} \mathrm{DS}_{2}$-VASc score.

3. However, IAS thickness could not independently distinguish high-risk patients who are prone to recurrence of arrhythmia before the atrial fibrillation ablation procedure.

\section{Conflict of interest}

No potential conflict of interest relevant to this article was reported.

\section{Acknowledgments}

We thank Sung Bok Lee, Byung Jin Choi, Seok Man Mun, and Eun Hee Choi for their valuable contributions to this study. This study was supported by grants (MD11004, ED15037) of the Korea University Medical Center.

\section{REFERENCES}

1. Wolf PA, Abbott RD, Kannel WB. Atrial fibrillation: a major contributor to stroke in the elderly. The Framingham Study. Arch Intern Med 1987;147:1561-1564.

2. Pappone C, Oreto G, Rosanio S, et al. Atrial electroanatomic remodeling after circumferential radiofrequency pulmonary vein ablation: efficacy of an anatomic approach in a large cohort of patients with atrial fibrillation. Circulation 2001;104:2539-2544.

3. Calkins H, Brugada J, Packer DL, et al. HRS/EHRA/ECAS expert consensus statement on catheter and surgical ablation of atrial fibrillation: recommendations for personnel, policy, procedures and follow-up. A report of the Heart Rhythm Society (HRS) Task Force on Catheter and Surgical Ablation of Atrial Fibrillation developed in partnership with the European Heart Rhythm Association (EHRA) and the European Cardiac Arrhythmia Society (ECAS); in collaboration with the American College of Cardiology (ACC), American Heart Association (AHA), and the Society of Thoracic Surgeons (STS). Endorsed and approved by the governing bodies of the American College of Cardiology, the American Heart Association, the European Cardiac Arrhythmia Society, the European Heart Rhythm Association, the Society of Thoracic Surgeons, and the Heart Rhythm Society. Europace 2007;9:335-379.

4. Ouyang F, Tilz R, Chun J, et al. Long-term results of catheter ablation in paroxysmal atrial fibrillation: lessons from a 5-year follow-up. Circulation 2010;122:2368-2377.

5. Weerasooriya R, Khairy P, Litalien J, et al. Catheter ablation for atrial fibrillation: are results maintained at 5 years of follow-up? J Am Coll Cardiol 2011;57:160-166.

6. Verma A, Wazni OM, Marrouche NF, et al. Pre-existent left atrial scarring in patients undergoing pulmonary vein antrum isolation: an independent predictor of procedural failure. J Am Coll Cardiol 2005;45:285-292.

7. Oakes RS, Badger TJ, Kholmovski EG, et al. Detection and quantification of left atrial structural remodeling with delayed-enhancement magnetic resonance imaging in 
patients with atrial fibrillation. Circulation 2009;119:17581767.

8. Chao TF, Cheng CC, Lin WS, et al. Associations among the $\operatorname{CHADS}(2)$ score, atrial substrate properties, and outcome of catheter ablation in patients with paroxysmal atrial fibrillation. Heart Rhythm 2011;8:1155-1159.

9. Kuppahally SS, Akoum N, Burgon NS, et al. Left atrial strain and strain rate in patients with paroxysmal and persistent atrial fibrillation: relationship to left atrial structural remodeling detected by delayed-enhancement MRI. Circ Cardiovasc Imaging 2010;3:231-239.

10. Lopez-Candales A. Is the presence of interatrial septal hypertrophy a marker for atrial fibrillation in the elderly? Am J Geriatr Cardiol 2002;11:399-403.

11. Lopez-Candales A, Grewal H, Katz W. The importance of increased interatrial septal thickness in patients with atrial fibrillation: a transesophageal echocardiographic study. Echocardiography 2005;22:408-414.

12. Shin SY, Yong HS, Lim HE, et al. Total and interatrial epicardial adipose tissues are independently associated with left atrial remodeling in patients with atrial fibrillation. J Cardiovasc Electrophysiol 2011;22:647-655.

13. Calkins H, Kuck KH, Cappato R, et al. 2012 HRS/EHRA/ ECAS expert consensus statement on catheter and surgical ablation of atrial fibrillation: recommendations for patient selection, procedural techniques, patient management and follow-up, definitions, endpoints, and research trial design: a report of the Heart Rhythm Society (HRS) Task Force on Catheter and Surgical Ablation of Atrial Fibrillation. Developed in partnership with the European Heart Rhythm Association (EHRA), a registered branch of the European Society of Cardiology (ESC) and the European Cardiac Arrhythmia Society (ECAS); and in collaboration with the American College of Cardiology (ACC), American Heart Association (AHA), the Asia Pacific Heart Rhythm Society (APHRS), and the Society of Thoracic Surgeons (STS). Endorsed by the governing bodies of the American College of Cardiology Foundation, the American Heart Association, the European Cardiac Arrhythmia Society, the European Heart Rhythm Association, the Society of Thoracic Surgeons, the Asia Pacific Heart Rhythm Society, and the Heart Rhythm Society. Heart Rhythm 2012;9:632-696.

14. Lang RM, Bierig M, Devereux RB, et al. Recommendations for chamber quantification. Eur J Echocardiogr 2006;7:79-108.
15. Na JO, Shin SY, Lim HE, et al. Impaired transport function of the left atrium and left atrial appendage in cryptogenic stroke patients with atrial septal aneurysm and without patent foramen ovale. Eur J Echocardiogr 2011;12:140-147.

16. Thanassoulis G, Massaro JM, O'Donnell CJ, et al. Pericardial fat is associated with prevalent atrial fibrillation: the Framingham Heart Study. Circ Arrhythm Electrophysiol 2010;3:345-350.

17. Al Chekakie MO, Welles CC, Metoyer R, et al. Pericardial fat is independently associated with human atrial fibrillation. J Am Coll Cardiol 2010;56:784-788.

18. Gay JD, Guileyardo JM, Townsend-Parchman JK, Ross K. Clinical and morphologic features of lipomatous hypertrophy ("massive fatty deposits") of the interatrial septum. Am J Forensic Med Pathol 1996;17:43-48.

19. Isner JM, Swan CS 2nd, Mikus JP, Carter BL. Lipomatous hypertrophy of the interatrial septum: in vivo diagnosis. Circulation 1982;66:470-473.

20. Devine WA, Anderson RH. Lipomatous hypertrophy ("massive fatty deposits") of the interatrial septum. Am J Forensic Med Pathol 1997;18:107-108.

21. Kluge WF. Lipomatous hypertrophy of the interatrial septum. Northwest Med 1969;68:25-30.

22. Hutter AM Jr, Page DL. Atrial arrhythmias and lipomatous hypertrophy of the cardiac interatrial septum. Am Heart J 1971;82:16-21.

23. Shirani J, Roberts WC. Clinical, electrocardiographic and morphologic features of massive fatty deposits ("lipomatous hypertrophy") in the atrial septum. J Am Coll Cardiol 1993;22:226-238.

24. Daccarett M, Badger TJ, Akoum N, et al. Association of left atrial fibrosis detected by delayed-enhancement magnetic resonance imaging and the risk of stroke in patients with atrial fibrillation. J Am Coll Cardiol 2011;57:831-838.

25. Chao TF, Lin YJ, Tsao HM, et al. CHADS(2) and CHA(2) DS(2)-VASc scores in the prediction of clinical outcomes in patients with atrial fibrillation after catheter ablation. J Am Coll Cardiol 2011;58:2380-2385.

26. European Heart Rhythm Association; European Association for Cardio-Thoracic Surgery, Camm AJ, Kirchhof P, et al. Guidelines for the management of atrial fibrillation: the Task Force for the Management of Atrial Fibrillation of the European Society of Cardiology (ESC). Eur Heart J 2010;31:2369-2429.

27. Fatkin D, Kuchar DL, Thorburn CW, Feneley MP. Trans- 
esophageal echocardiography before and during direct current cardioversion of atrial fibrillation: evidence for "atrial stunning" as a mechanism of thromboembolic complications. J Am Coll Cardiol 1994;23:307-316.

28. Kostin S, Klein G, Szalay Z, Hein S, Bauer EP, Schaper J.
Structural correlate of atrial fibrillation in human patients. Cardiovasc Res 2002;54:361-379.

29. Lombardo RM, Reina C, Abrignani MG, Braschi A, De Castro S. Embolic stroke, sinus rhythm and left atrial mechanical function. Eur J Echocardiogr 2006;7:401-404. 